\title{
Using Feedback to Control Deadtime in the CDF Trigger System
}

\author{
Donatella Torretta \\ for the CDF Collaboration \\ Fermi National Accelerator Laboratory \\ Batavia, Illinois 60510
}

\begin{abstract}
The CDF experiment uses a three-level trigger system to select events produced during $p \bar{p}$ collisions. As the luminosity of the Tevatron accelerator falls by a factor of four over a 24 hour period, trigger selections are adjusted automatically in order to make full use of the data processing bandwidth. The selections are made to maximize high purity triggers and keep the deadtime as low as possible at any given luminosity thoughout the entire course of a run. We describe the algoritms used to obtain these goals and how the changing conditions are accounted for in the analysis of the data.
\end{abstract}

\section{INTRODUCTION}

The Tevatron is the highest energy collider currently in operation. It is located at the Fermi National Accelerator Laboratory in Batavia, IL. The Tevatron collides proton and antiproton beams of $980 \mathrm{Gev}$ each in two interaction points where the $\mathrm{CDF}$ and $\mathrm{D} 0$ experiments are currently taking data. The CDF experiment [ref. 1] is a multipurpose detector with a broad high energy physics program studying many interesting physics processes like:

- Top quark measurements

- Precision Electro-Weak studies

- Search for new phenomena

- B physics

- Search for Higgs boson

The cross sections of these processes vary by a factor of $10^{10}$, going from $50 \mathrm{mb}$ of inelastic $p \bar{p}$ collisions to a $7 \mathrm{pb}$ for $t \bar{t}$ and $0.1 \mathrm{pb}$ for a light Higgs.

The Tevatron provides collisions at a rate of $1.7 \mathrm{MHz}$. The average event size of CDF is about $250 \mathrm{kB}$. Clearly we cannot write or analize data at this rate. We need to scale down to a manageable rate of $40 \mathrm{MB} / \mathrm{s}$ to tape. So the trigger system is crucial and must reject $99.99 \%$ of beam crossing rate but keep the events of interest with high efficiency.

\section{A. CDF Trigger System - Deadtime}

1) CDF Trigger System: The CDF experiment employes a three-level trigger system [see fig. 2 for details] to select events and achieve an output rate of approximately $150 \mathrm{~Hz}$, the current limit to write on tape.

The Level 1 is implemented with a synchronous 42 stage pipeline hardware trigger with an accept rate up to $35 \mathrm{kHz}$. A Level 1 decision is returned to the front-end crates in about $5 \mu s e c$ after bunch crossing time. When an event is accepted at Level 1, it is moved to one of four Level 2 buffers. At the same

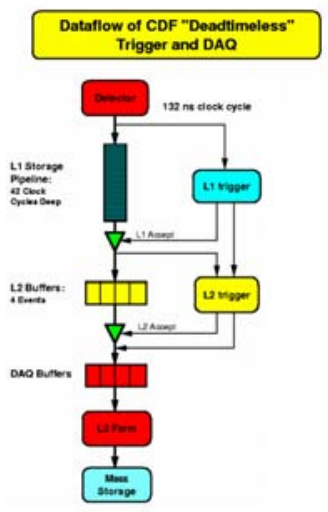

Fig. 1. Schematic view of the CDF trigger system. Deadtime occurs when none of the Level 2 buffers (yellow) is available

time the Level 1 information is sent to the Level 2 decision stage where some limited event reconstruction is performed and a Level 2 decision is taken. The Level 2 trigger operates asyncronously and is a combination of custom electronics for reconstruction and a cpu for decision (about $35 \mu \mathrm{sec}$ ) and an accept rate up to $800 \mathrm{~Hz}$.

When an event is accepted at Level 2, the corresponding Level 2 buffer is read out and the data are transferred to the Level 3 for a full event reconstruction. If the event is accepted by Level 3, is then written to tape with an output rate of about $40 \mathrm{MB} / \mathrm{s}$. The overall data rejection is therefore 1:10000.

2) Deadtime: If all four Level 2 buffers fill up, CDF will incurr in Deadtime. Infact, if all four buffers are full, it is not possible for the system to accept an otherwise good Level 1 trigger event: we refer to this as Deadtime. The deadtime has two primary sources:

- the product of (L1 Accept rate) * (L2 Processing time) is large

- the product of (L2 Accept rate) * (Readout time) is large

The Deadtime can be controlled adjusting trigger rates to stay within the DAQ limits. The CDF collaboration's goal is to keep deadtime below $5 \%$.

In addition to selection based on quantities such as calorimeter energy or charged particle track moments in the trigger systems, we can limit the number of events from high rate processes accepting only a fraction of the events that pass the 


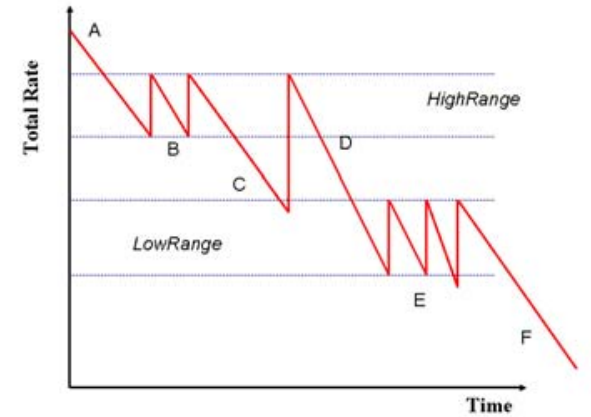

Fig. 2. Example behaviour of dynamic prescaling: A-F refer to the stages of running described in the text.

selection criteria: this is called a Prescale of $N$.

3) Luminosity, Trigger Rates and Deadtime: Luminosity is a measure of the beam intensity. As collisions remove particles from the beams, the luminosity decreases with time [ see fig. $3]$. As the luminosity drops, if we do nothing, the trigger rate also decreases and eventually there will be very small rates of interesting processes to be written on tape. On the other hand, the rates decrease, more data acquisition bandwidth becomes available. To make use this available bandwidth at all times CDF relies on a special system to relax trigger selection criteria during data taking so that we improve physics yield while keeping the total Level 1 and Level2 trigger rates stable.

This method is called Dynamic Prescaling.

\section{B. Dynamic Prescaling}

Dynamic Prescaling is used for both Level 1 and Level 2 trigger selections. The adjustments of dynamic prescales follows rules that take into account the total and individual trigger rates. Both Level 1 and Level 2 dynamically prescaled triggers are controlled by parameters that define a Maximum, a Default and a Minumum prescale values that are stored in an Oracle Database, along with the trigger cut values.

Two trigger rate ranges of operation, High and Low Range, are defined for both Level 1 and Level 2. A Java program adjusts the prescale values to keep the total rate in one of those target ranges. Changes happen based on rate information accumulated on a time scale of minutes and the amount of the prescale change depends on the available bandwidth available.

Fig. 2 shows the behavior of the total trigger rate (for either Level 1 or Level 2) as a function of time.

The following steps of operation can be seen:

A) At the beginning of the colliding period ( called store) the prescales are set to their Maximum values.

B) The total trigger rate starts decreasing, and when it reaches the lower end of the High range, the prescales are lowered from Maximum toward Default to set the total rate to the target of upper end of High range.

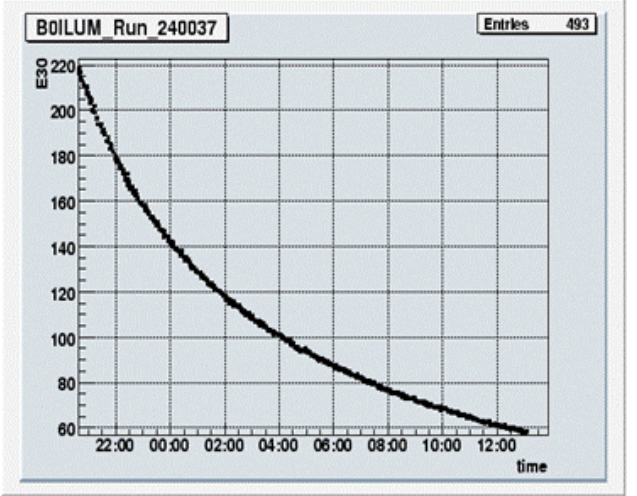

Fig. 3. Luminosity vs Time for a recent Tevatron store. Luminosity falls by a factor of 4 over 16 hours

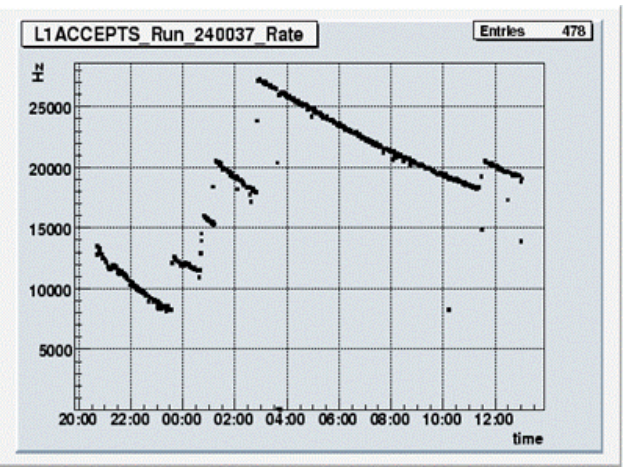

Fig. 4. L1 Accept Rate vs Time: rate increases due to Dynamic Prescaling are clearly visible

C) The process repeats until all prescales are at their Default values.

D) All prescales are at Default values.

E) When the rate reaches the lower limit of the Low range, the prescales are adjusted from Default towards Minimun.

F) When all prescales are at Minimum, no more prescale changes will take place, and the data taking will continue in these conditions until the store ends.

Fig. 4 and 5 show, respectively, the Level 1 Accept and Level 2 Accept rates versus time where are clearly visible the rate's increases due to the dynamic prescaling. Most noticeable in fig. 5 is the steady L2 rate for the whole data taking period, despite the luminosity falling of a factor of four. Fig. 6 shows the Deadtime for the same period of data taking.

\section{Prescale Accounting}

Many data analyses measure rates of physics processes, or cross sections.

Since the cross section of a physics process is constant but the background (other processes' signals that can simulate it) grows with increasing luminosity, we cannot simply calculate the prescale efficiency as simple ratio of totals in a given run.

We need to evaluate the "effective integrated luminosity" per each prescaled trigger. In order to do this we take advantege of the fact that the data collected is divided in small units, called 


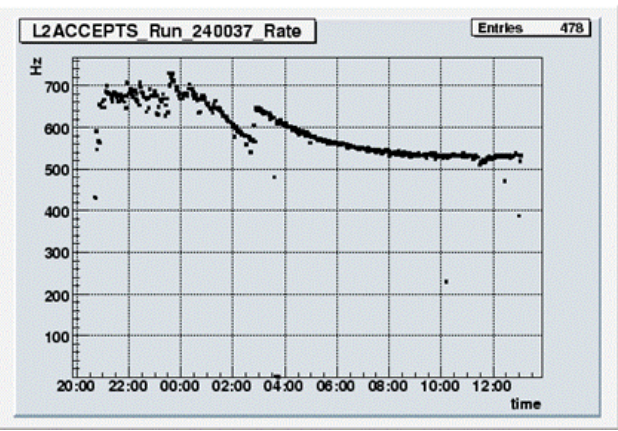

Fig. 5. L2 Accept Rate vs Time: rate steady across the whole data taking period

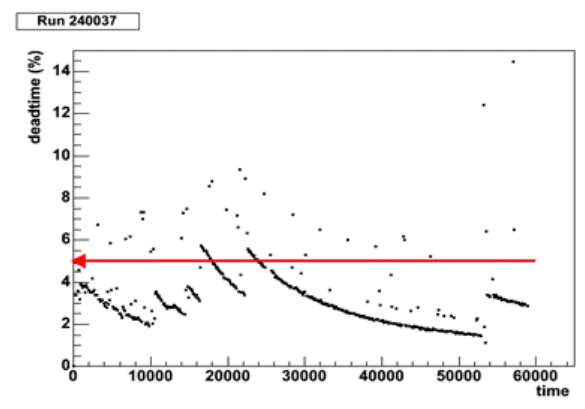

Fig. 6. Deadtime vs Time: in average, below $5 \%$

"run sections", which are short compared to the changing operating conditions. Therefore, in each run section, the luminosity changes are so small the luminosity can be considered constant. For each run sections, the number of events Before $\left(B_{i}\right)$ and After $\left(A_{i}\right)$ prescale is recorded for each trigger. We can then perform numeric integration by summing ober run sections to get the effective luminosity for each trigger

$$
\int L_{e f f}=\sum_{i}\left(A_{i} / B_{i}\right)\left(\int L d t\right)_{i}
$$

This information is the recorded in an Oracle Database to be used in physics data analysis.

\section{CONCLUSIONS}

The CDF experiment uses powerful techniques to control trigger rates and deadtime during data taking. Releasing trigger prescales maximizes the physics content on tape and the feedback based on rates gives operational stability, since the system responds automatically to changes in conditions for both indivisual and total trigger rates. The changed trigger conditions are accounted for in the analysis of the data.

\section{ACKNOWLEDGMENT}

The author would like to thank the whole CDF collaboration and especially the DAQ/Trigger groups for their input and support.

\section{REFERENCES}

[1] D. Acosta, et al., (CDF collaboration), Phys. Rev. D71, 032001 (2005).

[2] R.Blair et al. (CDFII Collaboration) The CDF runII Detector Technical Design Report, 1996, FERMILAB-Pub-96-390-E 